\title{
Face-to-Face vs. Digital Communication: The Effect of Communication Channels at the Point of Sale-An Abstract
}

\author{
Andreas Kessenbrock, Sören Köcher, and Hartmut Holzmüller
}

\begin{abstract}
Mobile devices provide retailers the opportunity to communicate and interact with their customers via a new channel, regardless of the customer's current whereabouts. Generally, mobile devices are used for a variety of reasons at the point of sale (Cliquet et al. 2014). Especially with regard to reduced search costs, customers can search the mobile web or use smartphone applications to quickly gather external information about various products before making a purchase decision (Daurer et al. 2015).

The intention of this research is to improve the knowledge of the impact of mobile devices as a communication channel in contrast to personal communication in a brick-and-mortar environment. Specifically, we investigate the impact of the two most frequent information sources, namely, salesperson and previous customers.

Contrary to a large number of studies which investigate the influence of recommendations on customers' behavior in isolation, this study provides an approach to examine the effects of communication channels and information source simultaneously. More precisely, this research offers an initial attempt to understand the effects of mobile devices as a new communication channel on customers' perceptions of recommendation persuasiveness and the importance of customers' perceived control of the communication process at the point of sale.

Despite retailers' quest to increase their understanding about the impact of new communication channels on customers' purchase decisions, this stream of research has been previously neglected. Across two experiments we show contradictory effects of digital communication and reveal that mobile devices (when compared to personal communication) enhance perceived control of the communication process. In this context, the study contributes new aspects to existing research on in-store communication while taking contemporary communication structures into account.
\end{abstract}

References available upon request.

\footnotetext{
A. Kessenbrock $(\varangle) \bullet$ S. Köcher $\bullet$ H. Holzmüller

TU Dortmund University, Dortmund 44227, Germany

e-mail: andreas.kessenbrock@tu-dortmund.de; soeren.koecher@tu-dortmund.de;

hartmut.holzmueller@tu-dortmund.de
} 